\title{
Show-through Watermarking of Duplex Printed Documents
}

\author{
Gaurav Sharma ${ }^{a}$ and Shen-ge Wang $^{b}$ \\ ${ }^{a}$ ECE Dept, Univ. of Rochester, Rochester, NY 14627-0126, USA; \\ ${ }^{b}$ Xerox Corporation, 800 Phillips Road, Webster, NY 14580
}

\begin{abstract}
A technique for watermarking duplex printed pages is presented. The technique produces visible watermark patterns like conventional watermarks embedded in paper fabric. Watermark information is embedded in halftones used to print images on either side. The watermark pattern is imperceptible when images printed on either side are viewed independently but becomes visible when the sheet of paper is held up against a light. The technique employs clustered dot halftones and embeds the watermark pattern as controlled local phase variations. Illumination with a back-light superimposes the halftone patterns on the two sides. Regions where the front and back-side halftones are in phase agreement appear lighter in show-through viewing, whereas regions over which the front and back side halftones are in phase disagreement appear darker. The image printed on one side has a controlled variation of the halftone phase and the one printed on the other side provides a constant phase reference. The watermark pattern is revealed when the sheet is viewed in "show-through mode" superimposing the halftones on the two sides. Threshold arrays for the halftone screens are designed to allow incorporation of a variety of halftone patterns while minimizing artifacts in images printed using these halftones.
\end{abstract}

Keywords: watermarking, hardcopy watermarking, halftoning, data embedding

\section{INTRODUCTION}

Traditional watermarks are visual patterns embedded in a sheet of paper. These watermarks are produced by creating a variation in the thickness of paper fiber during the wet-phase of paper making. The embedded design is imperceptible under normal viewing of either side but when the paper is held up to a light source the visual pattern is clearly visible. Techniques for producing these watermarks have been known since the late thirteenth century. ${ }^{1}$ Even today these are commonly used in currency, legal documents, and identification cards to make counterfeiting more difficult. In addition, they find use in special stationery for purposes of ornamentation.

Recent years have seen a significant research effort in digital watermarking and data hiding techniques. ${ }^{2-5}$ Unlike traditional watermarks, a majority of these techniques focus on embedding and extracting data from electronic content and not from a hardcopy paper document. A (smaller) number of techniques for embedding visual or non-visual data in hardcopy documents have also been proposed. Typically, the techniques are specific to the type of document and its content. Examples of this class of techniques include methods that embed information in hardcopy text documents through minor formatting and layout modifications, ${ }^{6}$ methods for embedding information in printed halftone images, ${ }^{7-11}$ methods for embedding information in images that are designed to be robust to the printing process ${ }^{12,13}$ and methods for joint watermarking and embedding. ${ }^{14}$ Usually these techniques require a capture of the printed document in electronic form from which the embedded data may be recovered or the visible pattern may be revealed through suitable processing.

In this paper, we propose a method for mimicking traditional watermarks for duplex printing of images on plain paper. Just like conventional watermarks that are embedded in the fabric of paper during manufacturing, the watermark pattern is not perceptible when images printed on either side are viewed independently but becomes visible when the sheet of paper is held up against the light. The watermark information is embedded in the halftones used to print images on either side. The proposed technique employs clustered dot halftones and embeds the watermark pattern as a controlled local phase variation in the halftones. When the sheet of paper is

Further author information: (Send correspondence to G. Sharma/S. Wang)

G.S.: E-mail: gsharma@ece.rochester.edu, Telephone: 15852757313

S.W.: E-mail: swang@crt.xerox.com, Telephone: 15854222887 
illuminated with a back-light, the halftones on the two sides are optically superimposed. Regions where the front and back-side halftones are in phase agreement appear lighter in show-through viewing, whereas regions over which the front and back side halftones are in phase disagreement appear darker. A chosen pattern is embedded as a controlled variation of the halftone phase of the image printed on one side. The halftone image printed on the other side provides a constant phase reference and the watermark pattern is revealed when the sheet is viewed in "show-through mode" superimposing the halftones on the two sides.

The proposed method is related to and builds upon techniques proposed earlier for visual embedding of data in halftone images. ${ }^{7,8}$ The algorithms proposed here and those proposed earlier for halftone information embedding both embed information in the phase correlation of halftones in different image regions (or images on different sides). The techniques, however, also have very significant differences. For techniques proposed in prior work, the watermark pattern is detected from a scan of the printed image (at a sufficiently high resolution). These methods therefore tend to rely on dispersed dot halftoning schemes, which offer significant freedom in the placement of individual halftone dots - thereby affording considerable flexibility in the phase correlation across different regions (i.e. embedding capacity). For the techniques proposed in this paper, the detection process is visual and involves viewing the printed sheet with a back-light. Since light undergoes considerable blurring in the passage through paper and because front to back registration in duplex printing can have significant phase errors, dispersed dot halftones cannot be used for this purpose on typical substrates. Clustered dot halftones are therefore used along with an embedding technique that reveals the pattern even in the presence of slow registration variations over the two sides.

\section{WATERMARK PATTERN EMBEDDING IN HALFTONE DOT CORRELATION}

Digital halftoning converts a continuous tone image to a bi-level image while attempting to preserve its visual appearance. Halftoning works by exploiting the low-pass characteristics of the human visual system. Ideally, a local spatial average of the bi-level image generated by the halftoning process at each spatial location closely approximates the original continuous tone image. There are many different halftoning algorithms adapted to various printing technologies and applications. ${ }^{15-18}$

Two common ways of classifying halftoning algorithms are based on their computational complexity and visual appearance. Along the axis of increasing computational complexity, halftone algorithms may be classified as screening methods or spatially adaptive methods. Screening algorithms generate the halftone image through a process of pixel by pixel comparison against a screen of threshold values (which are tiled to cover the image). Due to the simplicity of the screening process it is commonly preferred in low-cost or high speed printing systems. For spatially adaptive algorithms, the processing of each pixel also involves its spatial neighborhood. This usually offers significant improvements in quality, though at the cost of a much higher computational load. Common examples of spatially adaptive techniques include error-diffusion and model-based halftoning methods. On the basis of visual appearance, halftone techniques are typically classified as dispersed or clustered dot techniques. Dispersed dot techniques produce halftones with isolated individual pixel "dots" at the printer resolution. Different gray levels are achieved by varying the density of the dots. Clustered dot techniques produce halftones where pixels at the printer resolution cluster together to produce a printed halftone dot. Variation in gray level is achieved by changing the size of the cluster of pixels corresponding to each halftone dot. Clustered dot halftones are commonly employed in laser printing applications because the isolated single pixel dots from dispersed dot halftones do not reproduce well on these printers. They are also the primary halftones for lithographic printing and closely resemble conventional analog halftones. Dispersed dot halftones are commonly used in inkjet printing because they reproduce well on these devices.

Several methods have been proposed for embedding data in halftone images generated using dispersed dot halftoning methods..$^{7-10,14}$ Since there are a number of choices for the actual halftone pattern at each gray level that provide satisfactory visual performance (i.e., reasonably small energy at low spatial frequencies), data can be embedded as correlations among the halftone dots. This flexibility is exploited in several of the schemes. A specific concept ${ }^{7}$ is illustrated in Fig. 1 where the two squares represent regions of the image over which the halftone pattern is embedded as a correlation in the halftone dots. The halftone dots at corresponding pixels of the two square regions are "negatively correlated" over the regions labeled $W$ and $W$ ' and "positively correlated" over the remainder of the two squares (the regions labeled $S$ and $S^{\prime}$ ). That is, if a pixel in the region labeled $W$ 


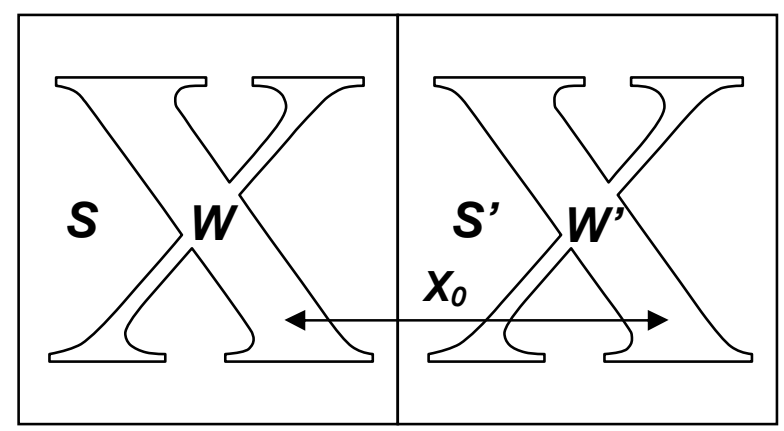

Figure 1. Illustration of data embedding in dispersed dot halftones using correlation among dots in different regions.

in the left square is white (black) in the binary halftone image, the corresponding pixel in the region labeled $W^{\prime}$ in the square to the right is more likely to be black (white). Similarly, if a pixel in the region labeled $S$ is white (black) in the halftone, corresponding pixel in the region labeled $S$ ' in the square to the right is more likely to be white (black). When the halftones in the two regions are overlaid (in good alignment) the halftone dots in the positively correlated regions will tend to overlap more frequently than the dots in the negatively correlated regions. Thus in the overlap, the region labeled $W$ will appear darker than the region labeled $S$ creating a visually distinguishable halftone pattern in the form of the " $\mathrm{X}$ " shaped region. The method for introducing the correlation in the halftone dots depends on the method used for halftoning. For halftones generated using a stochastic screen, a static pattern may be embedded by designing a halftone screen of size corresponding to the two squares, with suitable constraints imposed during the design process to enforce the desired correlation. ${ }^{8}$ For error diffusion, threshold modulation ${ }^{19}$ can be used to introduce the desired positive and negative correlation in a pattern that can be dynamically chosen at the time of halftoning. ${ }^{20}$

\subsection{Watermark Detection}

For halftone images that are available digitally, the process of overlaying regions to reveal the watermark pattern can be readily accomplished through a shift and logical "AND" of the image with itself, where the shift is selected to align the two squares used for embedding the watermark pattern. However, in most practical systems, the halftoning is performed just prior to printing and therefore detection must be based on the printed image. This poses significant additional challenges. The printed halftone image must be scanned with sufficient resolution to resolve the halftone dots and the detection must then be performed using the scanned image. Since the printing and scanning processes can introduce significant nonlinear and locally varying distortions, the process may require a locally varying alignment in order to obtain good detection performance. Designs based on conjugate halftone stochastic screens ${ }^{8}$ offer a particular advantage in this aspect because the screens are identical over the regions labeled $S$ and $S$ '. Typically, this region occupies a much large fraction of the total space than the the watermark pattern region (labeled $W$ and $W^{\prime}$ ). The optimal shift in order to maximize the visibility of the watermark pattern can therefore be determined as the shift for which the autocorrelation of the scanned image with itself is maximized, where the search is conducted over shifts in the neighborhood of the ideal value computed from the imaging parameters such as the printing and scanning resolution, and the halftone screen size. This allows "synchronization" of the watermark detector in a manner similar to other digital image watermarking schemes that use repetitive embedding of the watermark for synchronization and redundancy. ${ }^{21}$

\section{SHOW-THROUGH WATERMARKING}

As outlined above, considerable computational effort is required in the detection of watermarks embedded as halftone correlations even though the final detection typically involves visual recognition of the embedded pattern. For a duplex printed page, if the sheet of paper is viewed against a back-light, the images on the front and back sides are naturally overlaid and the detection may therefore be significantly simplified. This paper illustrates how such a scheme for show-through watermarking can be practically realized. 
In theory, one may conceive a very simple process for achieving show-through watermarking from existing halftone watermarking schemes as follows. The halftone image with an embedded watermark pattern in the form of correlation among halftone dots is printed on both sides with a relative shift between the two sides corresponding to the watermark embedding. When viewing against a back-light, the images on the two sides are optically overlaid revealing the embedded watermark pattern. In practice however, light undergoes significant blurring in the process of transmission through the paper. As a result, the image from the side with the backlighting is significantly blurred before it is optically overlaid on the image on the front side. For typical paper substrates and the dispersed dot halftone watermark embedding schemes mentioned in the last section, the blurring of light in transmission through the paper eliminates the high frequency halftone structure. As a result the embedded watermark pattern is not visible when a watermark bearing duplex page is viewed with a backlight. Alternative schemes for embedding are therefore necessary for watermark embedding for show-through viewing.

For the same device resolution, typical clustered dot halftones have larger printed dots than dispersed dot halftones. Thus the printed dot structure has substantial low frequency content. This low frequency content survives the blurring encountered in the process of transmission through paper and therefore offers an opportunity that may be exploited for show-through watermarking. Most clustered dot halftones used in the digital printing industry are implemented as halftone screens that are typically designed and hand-tuned by experts through a trial and error process. Thus, the techniques for halftone data embedding developed for dispersed dot halftoning schemes are not applicable to clustered dot halftones. Since the growth of the halftone dots occurs in clusters, there is very limited flexibility in the placement of halftone dot pixel elements. In addition, reliable detection of differences in pixel element placement for similar sized halftone dots is typically not feasible. Thus embedding of information in the halftone pixel placement is not appropriate for clustered dot halftones.

\subsection{Phase-Shift Embedding for Show-through Watermarking}

Local variations in phase and frequency offer an opportunity for embedding information in clustered dot halftones. Typical clustered dot halftones have a constant frequency and phase. For a spatially uniform input image * which has a constant value $g$ over the spatial dimensions, the halftone output is a periodic pattern which can be expressed as

$$
O_{g}(\mathbf{x})=\sum_{\mathbf{n}} D_{g}(\mathbf{x}-\mathbf{P n})
$$

where $\mathbf{x}=\left[x_{1}, x_{2}\right]$ denotes the spatial location, $\mathbf{P}=\left[\mathbf{p}_{1} \mathbf{p}_{2}\right]$ is the halftone periodicity matrix, $\mathbf{p}_{1}$ and $\mathbf{p}_{2}$ are the basis defining the halftone periodicity, $D_{g}(\cdot)$ represents the (binary) spatial profile for a single halftone dot for the gray level $g$ defined over a halftone cell $\mathcal{C}$ of area $\operatorname{det}(\mathbf{P})$.

Figure 2 illustrates the halftone pattern for a typical clustered dot halftone at a $50 \%$ gray level. The squares represent the halftone cells and the dark diamond shaped region in the center represents the halftone dot. Vectors $\mathbf{p}_{1}$ and $\mathbf{p}_{2}$ that define the two-dimensional periodicity of the halftone are also illustrated in the figure.

The principle of embedding a watermark pattern using a variation in phase of the clustered dot halftone is illustrated in Figs. 3 and 4. Fig. 3 illustrates the overlap of two halftoned regions both at a $50 \%$ gray level and in phase agreement with each other. Since the corresponding dark and light regions of the two halftones overlap, the overlap is the same as either of the halftone patterns and also corresponds to a $50 \%$ gray level. Fig. 4 illustrates the overlap of two clustered dot halftones at a $50 \%$ gray level that are out of phase with each other. In this case, the overlap is dark everywhere and therefore significantly darker than either of the halftones that are overlapped. Note that a shift of "half" the halftone period produces the out of phase relation. Thus the halftone that is out of phase with with halftone of Eqn. (1) is given by

$$
\bar{O}_{g}(\mathbf{x})=O_{g}\left(\mathbf{x}-\frac{\mathbf{p}_{1}+\mathbf{p}_{2}}{2}\right)
$$

\footnotetext{
${ }^{*}$ For simplicity and clarity of presentation, we develop our discussion based on uniform images. The arguments are readily extended to halftones of actual spatially varying images.
} 


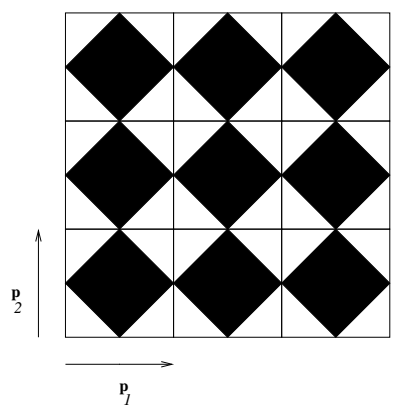

Figure 2. Clustered dot halftone (0/90 deg orientation) at a $50 \%$ gray level.
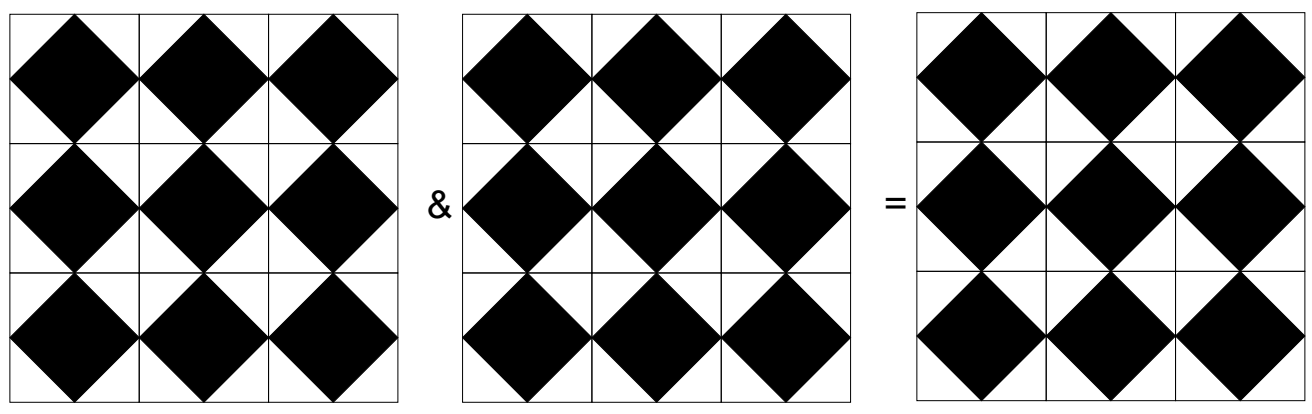

Figure 3. Overlap of in phase clustered dot halftones at a 50\% gray level.
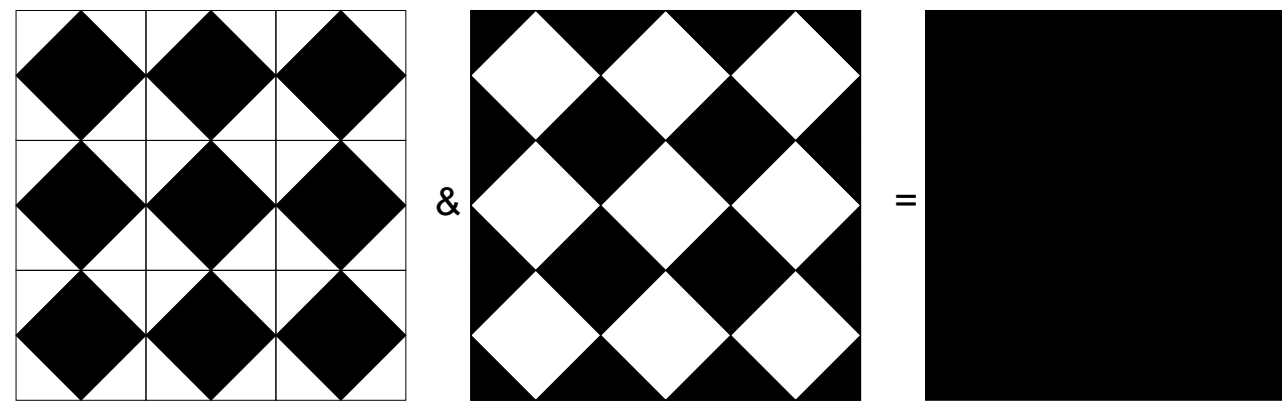

Figure 4. Overlap of out of phase clustered dot halftones at a $50 \%$ gray level. 
From this illustration it is obvious that variation in phase of a clustered dot halftone may be used to embed a visible watermark pattern in the overlap of two clustered dot halftone images. Regions over which the overlapped halftones are in phase agreement will appear lighter and regions over which the overlapped halftones are in phase dis-agreement will appear darker. By suitably controlling the phase relation, a desired pattern may be embedded.

Since clustered dots are much larger than the individual printer pixels, the blurring during the propagation of light through paper has a lower impact on the relative phase between the clustered dot halftones. The process is therefore suitable for show-through watermarking. We next outline how a suitable halftone screen can be created for the watermark embedding.

\subsection{Phase-Shift Clustered Dot Screens}

Note that the half period shift required for changing the phase relation of the two clustered dot halftones in our example of the previous section can also be expressed as a "circular" shift of the function $D_{g}(\cdot)$ representing the dot spatial profile. Thus Eqn. 2 can be rewritten as

$$
\begin{aligned}
\bar{O}_{g}(\mathbf{x}) & =O_{g}\left(\mathbf{x}-\frac{\mathbf{p}_{1}+\mathbf{p}_{2}}{2}\right) \\
& =\sum_{\mathbf{n}} D_{g}\left(\mathbf{x}-\mathbf{P n}-\frac{\mathbf{p}_{1}+\mathbf{p}_{2}}{2}\right) \\
& =\sum_{\mathbf{n}} \bar{D}_{g}(\mathbf{x}-\mathbf{P n})
\end{aligned}
$$

where $\bar{D}_{g}(\cdot)$ represents a two-dimensional "circular" shift of the function $D_{g}(\cdot)$ by the vector $\frac{\mathbf{p}_{1}+\mathbf{p}_{2}}{2}$ corresponding to half the period of the halftone. The term "circular" shift in this context is understood to correspond to the halftone cell.

Eqn. 3 indicates that the phase shift required for embedding of the watermark may equivalently be incorporated in the halftone dot profile $\bar{D}_{g}(\cdot)$ or correspondingly in the screen of halftone thresholds. In order to produce a halftone screen with an embedded watermark pattern, a large tile consisting of multiple halftone dots is designed. The visible pattern to be embedded is built into the tile by incorporating an appropriate phase shift in the halftone dots. We illustrate this by means of an example. Consider a region from a sample tile as shown in Fig. 5 where the squares represent the halftone cells as they would be in the absence of any phase shift. The centers of the black dots represent the centers of halftone dots in the presence of the phase shift. As one moves across halftone cells from left to right in Fig. 5, the halftones undergo a phase shift, or equivalently the center of the halftone dots moves in relation to the "cell". Over the course of five halftone cells in the horizontal direction, the center of the halftone dot undergoes a shift corresponding to half a halftone cell $\left(\frac{\mathbf{p}_{1}+\mathbf{p}_{2}}{2}\right)$ over the next five halftone cells this phase shift is reversed (so that tiling of the plane does not result in boundaries). Consider the overlap of halftones generated with this embedded phase shift with an independent halftone pattern with no phase shift and aligned with the square halftone cell boundaries indicated in the figure. Along the left vertical edge, the two halftones are in phase and the overlap will therefore appear lighter for most gray levels. Along the vertical line at a distance of five halftone cells from the left edge the halftones are completely out of phase and the overlap will therefore produce a darker region. Since the phase is entirely dependent on the horizontal displacement, alternate light and dark vertical lines will be seen in the overlap. Since the dots are much larger than the individual pixel dots in dispersed dot halftones, the pattern is also visible when the two halftones are printed on two sides of a sheet of paper and the overlay is achieved by viewing with a back-light.

Once the centers of the halftone dots have been determined (as in the above example), a screen incorporating the desired phase shift can be designed, for instance, as a Stoclustic halftone screen. ${ }^{22}$ We present a brief outline of the process. The screen covers the region corresponding to the multiple "halftone cells". Each pixel location in the screen is allocated a threshold value, which we assume is unique. Thus the screen is equivalently specified by the sequence $\mathcal{S}=\left\{\left(x_{i}, y_{i}\right)\right\}_{i=i}^{N}$ of pixel locations, where $\left(x_{i}, y_{i}\right)$ is the location corresponding to threshold $i$ and $N$ is the total number of pixels in the screen. In order to produce the screen with the tightest clustered dots, the sequence $\left\{\left(x_{i}, y_{i}\right)\right\}_{i=i}^{N}$ is selected so as to minimize the second moment of the pixels constituting the printed dot at each threshold from 1 to $N$ about the center pixel for the closest center (whose coordinates in general 


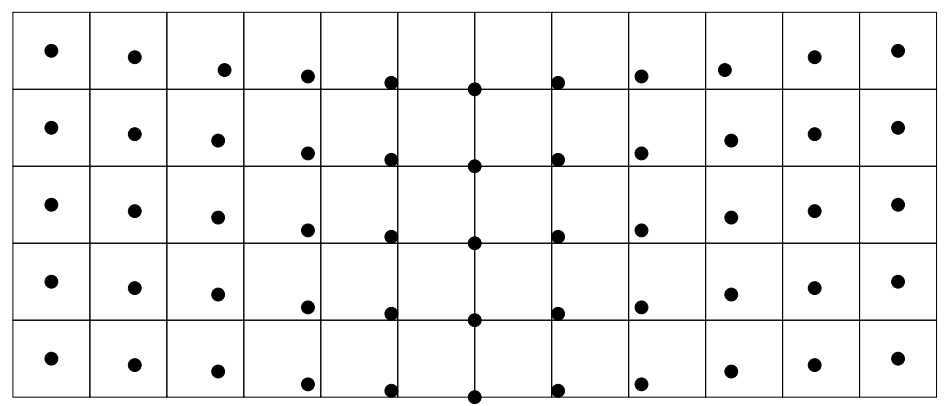

Figure 5. Illustration of the transition region of a threshold array incorporating a half period phase shift.

do not correspond to an integral number of pixels). Thus the screen is designed by minimizing the objective function

$$
\phi(\mathcal{S})=\sum_{g=1}^{N} \mu_{g}(\mathcal{S})
$$

where

$$
\mu_{g}(\mathcal{S})= \begin{cases}\sum_{i=1}^{g}\left(x_{i}-x_{c i}\right)^{2}+\left(y_{i}-y_{c i}\right)^{2} & g \leq N / 2 \\ \sum_{i=g}^{N}\left(x_{i}-x_{c i}\right)^{2}+\left(y_{i}-y_{c i}\right)^{2} & g>N / 2\end{cases}
$$

where $\left(x_{c i}, y_{c i}\right)$ denotes the halftone dot center location closest to the pixel $x_{i}, y_{i}$. The sequence $\mathcal{S}=\left\{\left(x_{i}, y_{i}\right)\right\}_{i=i}^{N}$ represents a candidate choice for the halftone screen. The function $\mu_{g}(\mathcal{S})$ represents the second moment of the minority pixel elements that constitute the halftone dots at gray level $g$ about the closest halftone center. Thus the minimization of $\phi(\mathcal{S})$ produces halftone dots that are tightly clustered about each of the halftone centers. A stochastic optimization algorithm based on simulated annealing is utilized for the minimization.

The example presented above illustrated the creation of a watermark pattern in the form of alternate light and dark vertical lines. Through an appropriate choice of the phase shift alternate patterns can be embedded. In addition, a template of sub-elements that are used to form an overall watermark pattern may be created by designing a set of screens that tile together without boundary artifacts (i.e. are in phase at the edges of the screen). Watermark patterns can then be created through a combination of these individual elements. Halftone threshold screens can thus be designed to allow incorporation of a variety of watermark patterns while minimizing artifacts in images printed using these halftones.

It is also worth mentioning here that typical laser printers allow finer addressability along one direction than in the other. In particular, along the direction corresponding to the laser scan, a finer addressability (and often resolution) can be obtained by modulation of the laser power at a rate higher than the pixel elements. This is often referred to as high addressability. ${ }^{16}$ A typical high addressability is $8: 1$ corresponding to eight addressable elements per pixel element. High addressability is particularly useful in the design of watermark patterns through phase shift and can be exploited to improve the resolution of embedded watermark patterns by designing the halftone screen over the grid corresponding to high addressability pixels.

\section{EXPERIMENTAL RESULTS}

Clustered halftone screens were designed for embedding various patterns through the phase shift process described in previous sections. Images halftoned with these screens were printed on one side of a sheet of paper and images halftoned with a constant halftone phase were printed on the other side. The embedded watermark patterns can be clearly seen when such duplex prints are viewed against a back-light.

A clustered dot watermark screen was designed for embedding the Xerox "X" logo as a phase shift pattern using the method proposed in this paper. Figs. 6 and 7 illustrate the watermark. Figure 6 shows a clustered dot 
halftone image ${ }^{\dagger}$. Figure 7 illustrates the phase-shift watermark, where halftone image of Fig. 6 is overlaid with a halftone representation of the same contone image obtained by using the phase shift watermark screen. The distinctive " $\mathrm{X}$ " logo is corresponding to the watermark pattern is clearly visible in the overlap.

The watermark is also illustrated in the duplex printed sheet included as an insert in the hardcopy proceedings including this paper. The halftone pattern is not perceptible when either side of this duplex printed sheet is viewed. However, when the sheet is held up against a light the embedded "X" logo can be seen.

The embedding of another watermark pattern is illustrated in Fig. 8 which represents the scan of a duplex halftone print of a uniform region bearing a "T" shaped watermark pattern. The scan was performed in transparency mode (which corresponds to viewing with a backlight) and has been inverted and contrast enhanced to ensure that the pattern is not lost as a result of the printer/display tone response. The "T" shaped pattern is clearly visible in the scan. Also apparent is the phase shift. It is worth remarking here that the different "T" shaped patterns visible in the figure are either light or dark and may also appear to vary over a single "T". The primary reason for this is the distortion produced in the printing process and the variation in front to back registration over the page. Unless these variations are carefully controlled, there is a slow locally varying phase ambiguity between the front and back images. The watermark pattern is however still visible as a variation in the darkness when the print is viewed with a backlight.

\section{DISCUSSION}

As illustrated in the last section, show-through watermarks may be embedded in duplex printed documents using phase shifts in clustered dot halftones. Several observations can be made regarding the showthrough watermarking process.

- As a security feature show-through watermarking only provides limited security to the extent that typical users cannot arbitrarily control the halftoning process and the front to back registration on their printers. With access to this control and sufficient time to design appropriate screens, the security of such a system may be compromised. The same is however true for conventional watermarks, though the barrier against counterfeiting is significantly higher in that case because access to paper making equipment is not as easy to obtain.

- The visibility of the watermark pattern is dependent on the image gray level in the watermarked region. From the discussion in Section 3.1 it is apparent that the highest contrast for the watermark pattern is obtained when images printed on both sides are at roughly $50 \%$ halftone coverage. Very dark and light areas have little halftone structure and therefore do not offer much contrast between in-phase and out-of-phase alignment of halftones. This is also apparent in the examples presented in the previous section.

- For the examples presented here, the watermark pattern was embedded entirely in the image printed on one side and the image on the other side only provided a phase reference. Such a scheme has the advantage that the watermark pattern is visible even if the front to back registration and printing distortions vary from page to page and slowly across the page. If originals are printed on high quality equipment with very good registration and minimal distortions, the modulation in relative phase may be shared across the images on the two sides. This will reduce any visible artifacts and also provide additional security against typical copying equipment.

- The blurring of light in the paper substrate limits the resolution achievable in show-through watermarking. The spreading of light in paper is dependent on the substrate(see for instance ${ }^{23}$ for a comparison of two substrates). Thinner or more transparent substrates will allow finer features in the watermark pattern than thicker more opaque substrates.

- The embedding process was illustrated using threshold screens. By developing/modifying suitable adaptive techniques for clustered dot halftoning, the phase shift may be introduced dynamically allowing greater flexibility.

\footnotetext{
${ }^{\dagger}$ Scaling of this and other halftone images included in this paper for the purposes of printing in the proceedings or display may introduce unwanted visual artifacts not seen in the original images.
} 


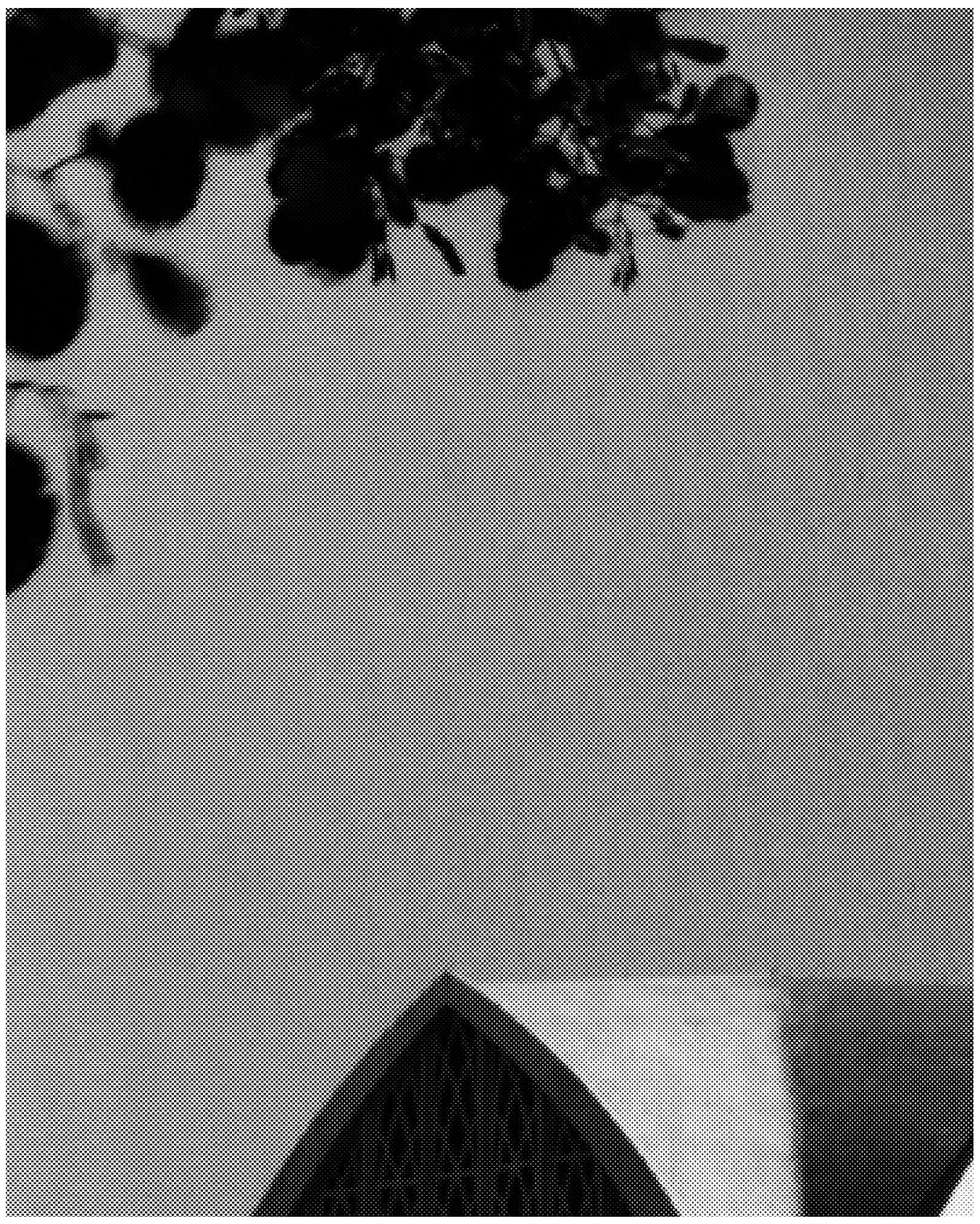

Figure 6. Halftoned image region. 


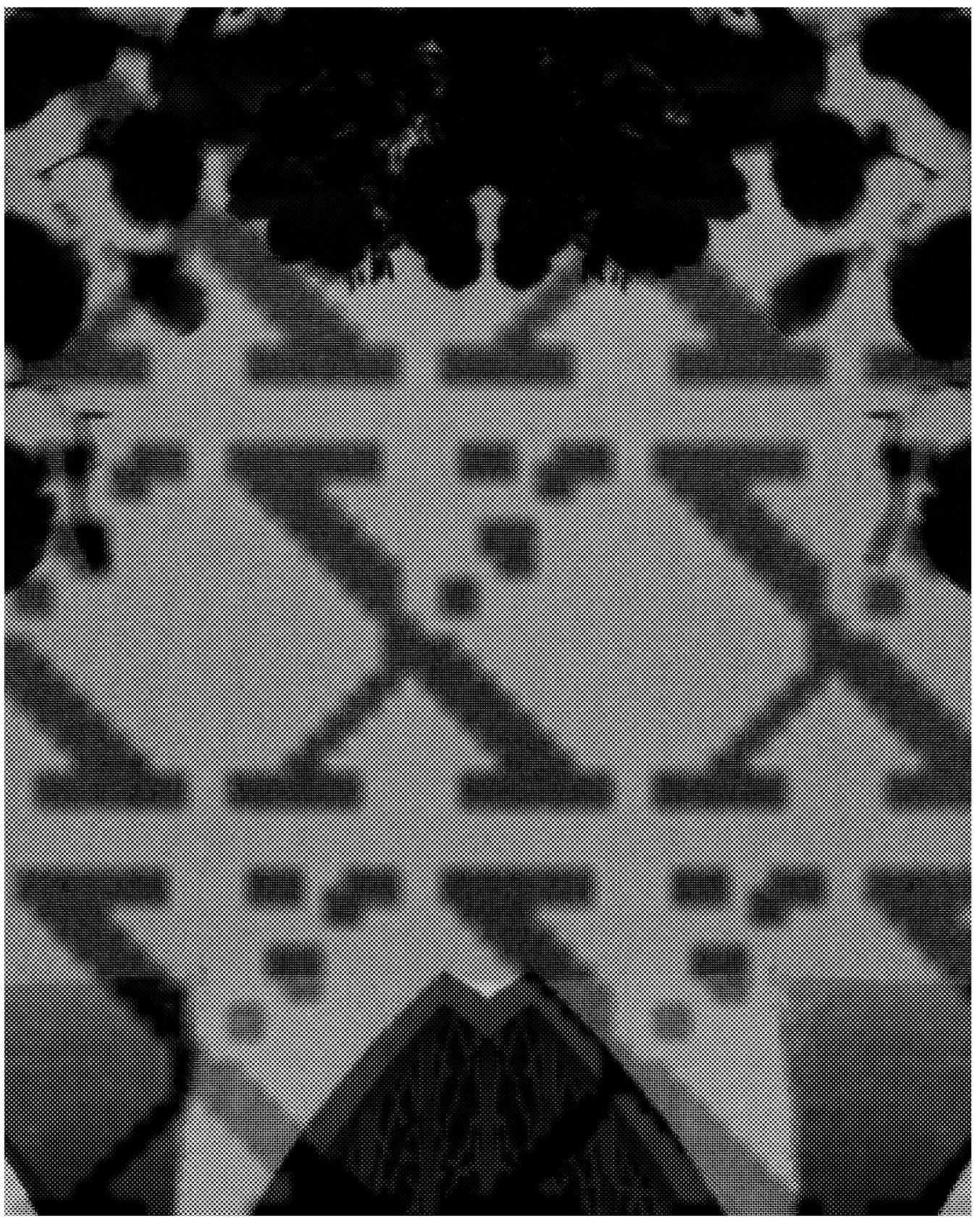

Figure 7. Electronic simulation of the overlap of halftone images printed on two sides showing watermark "X" pattern. 


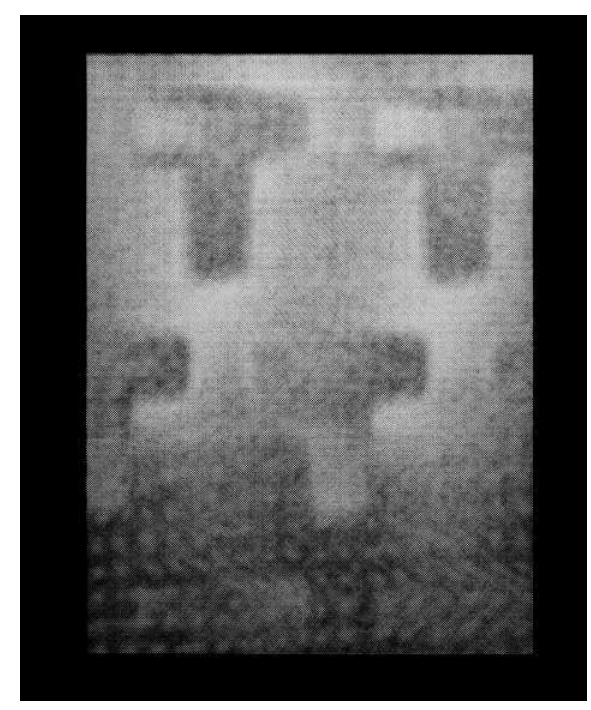

Figure 8. Transparency mode scan of a duplex print bearing a "T" shaped watermark pattern (after inversion and contrast enhancement).

\section{CONCLUSION}

In this paper, we illustrated how we may utilize suitably designed halftones to mimic traditional watermarks in duplex printed documents. The embedded watermark pattern is not visible when viewing the images printed on either side of the paper but is revealed when the sheet is viewed with a source of light placed behind it. The watermarks are embedded using a phase shift in clustered dot halftones and can be incorporated in halftone screens used to generate the halftones.

\section{REFERENCES}

1. "Encyclopaedia brittanica: Online edition." http://www.britannica.com/, 2003.

2. "Special issue on copyright and privacy protection," IEEE Trans.Selected Areas in Comm. 16, May 1998.

3. "Special issue on identification and protection of multimedia information," Proc. IEEE 87, Jul. 1999.

4. "Special issue on digital watermarking," IEEE Sig. Proc. Mag. 17, Sept. 2000.

5. C. I. Podilchuk and E. J. Delp, "Digital watermarking: algorithms and applications," IEEE Sig. Proc. Mag. 18, pp. 33-46, Jul. 2001.

6. J. Brassil, S. Low, M. N, and L. O'Gorman, "Electronic marking and identification techniques to discourage document copying," IEEE Trans.Selected Areas in Comm. 13, pp. 1495-1504, 1995.

7. K. T. Knox and S. Wang, "Digital watermarks using stochastic screens," in Proc. SPIE: Color Imaging: Device Independent Color, Color Hard Copy, and Graphic Arts II, G. B. Beretta and R. Eschbach, eds., 3018, pp. 316-322, Feb. 1997.

8. S. Wang and K. T. Knox, "Embedding digital watermarks in halftone screens," in Wong and Delp, ${ }^{24}$ pp. 218-227.

9. M. S. Fu and O. C. Au, "Data hiding for halftone images," in Wong and Delp, ${ }^{24}$ pp. $228-236$.

10. Z. Baharav and D. Shaked, "Watermarking of dither halftoned images," in Proc. SPIE: Security and Watermarking of Multimedia Contents, P. W. Wong and E. J. Delp, eds., 3657, pp. 307-316, Jan. 1999.

11. H. Z. Hel-Or, "Watermarking and copyright labeling of printed images," J. Electronic Imaging 10, pp. 794803, Jul. 2001.

12. A. M. Alattar, "Smart images using digimarc's watermarking technology," in Wong and Delp, ${ }^{24}$ pp. $264-273$.

13. S. Decker, "Engineering considerations in commercial watermarking," IEEE Comm. Mag. 39, pp. 128-133, Aug. 2001. 
14. D. Kacker and J. P. Allebach, "Joint halftoning and watermarking," in Proc. IEEE Intl. Conf. Image Proc., Sept. 2000.

15. J. P. Allebach, ed., Selected Papers on Digital Halftoning, vol. MS 154, SPIE, Bellingham, WA, 1999.

16. C. M. Hains, S. Wang, and K. T. Knox, "Digital color halftones," in Digital Color Imaging Handbook, G. Sharma, ed., CRC Press, Boca Raton, FL, 2003. Chapter 6.

17. H. R. Kang, Digital Color Halftoning, IEEE Press, Piscataway, NJ, 1999.

18. P. Roetling and R. P. Loce, "Digital halftoning," in Image Processing: Fundamentals and Applications, E. R. Dougherty, ed., Marcel Dekker, New York, NY, 1994. Chapter 10.

19. K. T. Knox and R. Eschbach, "Threshold modulation in error diffusion," J. Electronic Imaging 2, pp. 185192, Jul. 1993.

20. S. J. Harrington, "Method and apparatus for digital watermarking using error diffusion." United States Patent No. 6636616, 21 Oct. 2003.

21. C. W. Honsinger, P. W. Jones, M. Rabbani, and J. C. Stoffel, "Lossless recovery of an original image containing embedded data," US Pat. \#6,278,791, Aug. 2001.

22. S. Wang, "Stoclustic (stochastic clustered) halftone screen design," in Proc. ISEST's NIP 13: International Conference on Digital Printing Technologies, pp. 516-521, (Seattle, WA), Nov. 1997.

23. G. Sharma, "Show-through cancellation in scans of duplex printed documents," IEEE Trans. Image Proc. 10, pp. 736-754, May 2001.

24. P. W. Wong and E. J. Delp, eds., Proc. SPIE: Security and Watermarking of Multimedia Contents II, 3971, Jan. 2000. 